INPLASY

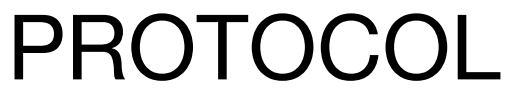

To cite: Xiong et al. Treatment of Ulcerative colitis with Chinese herbal medicine enema: a protocol for a systematic review and metaanalysis. Inplasy protocol 2020100091. doi:

10.37766/inplasy2020.10.0091

Received: 23 October 2020

Published: 23 October 2020

Corresponding author:

PeiYu Xiong

649716671@qq.com

Author Affiliation:

Chengdu University of

Traditional Chinese Medicine

Support: Sichuan Science and Technology.

Review Stage at time of this submission: The review has not yet started.

Conflicts of interest:

All authors involved in this work have no conflicts of interest.

\section{Treatment of Ulcerative colitis with Chinese herbal medicine enema: a protocol for a systematic review and meta-analysis}

Xiong, PY1; Ren, YH2; Zhong, C3; Zhang, PX4; Chen, X5; Liu, XL6; Bo, J7.

Review question / Objective: To evalutate the sfficacy and safety of Chinese herbal medicine enema in the treatment of ulcerative colitis.

Condition being studied: Ulcerative colitis (UC) is a chronic non-specific inflammatory disease of the rectum and colon whose etiology is not clear. In recent years, Chinese herbal medicine treatment of UC are more reports, and treatment methods varied. Enema therapy makes the drug directly act on the site of the disease, increases the local blood concentration in the intestine, and promotes mucosal healing. The efficacy of traditional Chinese medicine enema in the treatment of ulcerative colitis has been clinically proven, but there is a lack of systematic evaluation and meta-analysis. Our purpose is to evaluate the efficacy and safety of traditional Chinese medicine enema UC.

INPLASY registration number: This protocol was registered with the International Platform of Registered Systematic Review and Meta-Analysis Protocols (INPLASY) on 23 October 2020 and was last updated on 23 October 2020 (registration number INPLASY2020100091).

\section{INTRODUCTION}

Review question / Objective: To evalutate the sfficacy and safety of Chinese herbal medicine enema in the treatment of ulcerative colitis.
Condition being studied: Ulcerative colitis (UC) is a chronic non-specific inflammatory disease of the rectum and colon whose etiology is not clear. In recent years, Chinese herbal medicine treatment of UC are more reports, and treatment methods varied. Enema therapy makes the drug 
directly act on the site of the disease, increases the local blood concentration in the intestine, and promotes mucosal healing. The efficacy of traditional Chinese medicine enema in the treatment of ulcerative colitis has been clinically proven, but there is a lack of systematic evaluation and meta-analysis. Our purpose is to evaluate the efficacy and safety of traditional Chinese medicine enema UC.

\section{METHODS}

Search strategy: Adjust based on the following databases: CBM, CNKI, WF, VIP, Web of Science, Embase, PubMed, Cochrane Library, WHO ICTRP, ChiCTR, Clinical Trials, Grey Literature Database. No limitation on language or publication types restriction will be applied.

Participant or population: Patients with ulcerative colitis of different ages, genders, races, occupations or educational backgrounds can be included in the study. Exclude bacterial dysentery, amoebic colitis, intestinal tuberculosis, schistosomiasis, irritable bowel syndrome, ischemic colitis, Crohn's disease, radiation enteritis.

Intervention: The intervention measures included in the trial will be Chinese herbal medicine enema. The dose, duration and frequency of treatment are not limited.

Comparator: The control group includes placebo, blank control group and conventional drugs (such as sulfasalazine). The dose, time and frequency of treatment are not limited.

Study designs to be included: All relevant RCTs published in English and Chinese on Chinese herbal medicine enema for treatment of UC can be included. Nonrandomized controlled trials, reviews, case reports, experimental studies, expert experience and duplicate publications will be be excluded.

Eligibility criteria: The study only selected the clinical trials of Chinese herbal medicine enema in the treatment of UC.
Non-randomized controlled trials, reviews, case reports, experimental studies, expert experience and duplicate publications will be excluded.

Information sources: The database of randomized controlled trials related to Chinese herbal medicine enema treatment of UC was reviewed to collect the following: CBM, CNKI, WF, VIP, Web of Science, Embase, PubMed, Cochrane Library, WHO ICTRP, ChiCTR, Clinical Trials, Grey Literature Database. No limitation on language or publication types restriction will be applied.

Main outcome(s): Mainly observe the clinical efficacy. According to the consensus opinion of TCM experts on ulcerative colitis (2017), the efficacy evaluation criteria of TCM syndromes are divided into: clinical remission, marked effect, effective, and ineffective. Calculation formula: curative effect index $(\%)=$ (integral before treatment-integral after treatment) $\div$ integral before treatment $x$ $100 \%$.

Additional outcome(s): 1. Disease curative effect is divided into clinical curative effect (effective, relieved) and colonoscopy curative effect (endoscopic response, mucosal healing) for evaluation, using the modified Mayo activity index. 2. Mucosal histology score, using Geboes index. 3. Quality of life score: Refer to the IBD Questionnaire (IBDQ) for evaluation.5. The incidence of adverse events.

Data management: Two independent reviewers will extract data from eligible studies and enter the following information into the data extraction table. When a consensus cannot be reached on data extraction, the decision is made by the third author.

Quality assessment / Risk of bias analysis: Two researchers will independently conduct literature search, screening and data extraction. If there is a disagreement, the team should discuss and reach agreement. If necessary, third-party researchers will be invited to assist in 
judgment. The article will extract the following information: first author, country/ region, publication year, study design, follow-up, disease duration, sample size, detailed intervention, control treatment, etc. When the article data results are missing or ambiguous, one of the researchers will contact the original author to obtain detailed experimental data results.

Strategy of data synthesis: The metaanalysis will be performed using the software RevMan 5.3.If there is no statistic heterogeneity between study results, a fixed-effect model will be used for metaanalysis. However, if statistica heterogeneity exists, the source of heterogeneity should be further analyzed. In cases where significant clinical heterogeneity effects are excluded, a random-effects model will be used for meta-analysis.Meaningful heterogeneity will be explained by any other assessment, including data-dependent sensitivity analysis or subgroup analysis.

Subgroup analysis: If necessary, subgroup analysis will be performed based on detailed interventions, participant characteristics, and outcome measures.

Sensibility analysis: If the subgroup analysis is not satisfactory, we will use sensitivity analysis to assess the robustness of the results. The metaanalysis reorganized and merged the data, and finally compared with the previous results.

Language: English.

Country(ies) involved: China.

Keywords: Chinese herbal medicine enema, ulcerative colitis, systematic review, protocol.

Contributions of each author:

Author 1 - PeiYu Xiong.

Author 2 - Yuhang Ren.

Author 3 - Chun Zhong.

Author 4 - Peixu Zhang.

Author 5 - Xu Chen.
Author 6 - Xinglong Chen.

Author 7 - Bo Jia. 